\title{
Política, medios de comunicación y la enseñanza de la Estadística
}

\author{
Alejandro Galindo Alba ${ }^{(1)}$, Elena Misa Borrego ${ }^{(2)}$, Juan Núñez Valdés ${ }^{(3)}$ \\ (1) Departamento de Análisis Económico y Economía Política, Universidad de \\ Sevilla, Avda. Ramón y Cajal nº 1, alegalalb@gmail.com \\ (2) Departamento de Geometría y Topología, Universidad de Sevilla, Calle \\ Tarifa s/n, elena.misa.borrego@gmail.com \\ (3) Departamento de Geometría y Topología, Universidad de Sevilla, Calle \\ Tarifas/n,jnvaldes@us.es
}

Presentado en eXIDO16 (2016)

\begin{abstract}
RESUMEN
Los autores presentan una reflexión sobre la aplicación de contenidos en educación política y medios de comunicación a las unidades didácticas de Estadística durante la Educación Secundaria. Su objetivo es hacerle más interesante al alumnado dichos contenidos, que normalmente suelen ubicarse a final de curso y muchas veces se imparten de forma bastante superficial o incluso se omiten. Con ello se desea potenciar el trabajo en equipo, la investigación y la reflexión autónoma del alumno. Para este fin, proponemos una serie de actividades que faciliten el aprendizaje de los contenidos de esta rama de las Matemáticas, una de las más aplicadas en el mundo actual de las comunicaciones y cuyos contenidos básicos son necesarios para el correcto desarrollo de muchos estudios superiores de formación profesional y carreras universitarias. Se comienza mostrando la biografía de una de las mujeres matemáticas más relacionadas con la Estadística: Florence Nightingale. Seguidamente se establece una relación entre la Estadística y los medios de comunicación, tratando de llevar al alumno a la reflexión a través de errores encontrados tanto en prensa como en televisión. Finalmente, tratamos de acercar la Política al aula a través de su relación con la Estadística.
\end{abstract}

Palabras clave: Docencia en Estadística; cultura estadística; actividades relacionadas con la Estadística; política; medios de comunicación.

\section{INTRODUCCIÓN}

La Estadística está presente en la mayor parte de las actividades que nos rodean a pesar de que la mayoría no nos percatemos de ello. Desde que nos levantamos y encendemos la luz hasta en los detalles más insignificantes de nuestra vida cotidiana, como el nivel de consumo con tarjetas de crédito, el tiempo de espera en una llamada telefónica o quién ganará la liga, las técnicas estadísticas tienen un papel fundamental.

Esta realidad se puede aplicar a las unidades didácticas de Estadística de la E.S.O. y Bachillerato, no solo para hacer más atractivos los contenidos al 
alumnado, sino para evitar futuros errores en la vida adulta y contribuir a formar seres humanos más libres y responsables.

Nótese además que, en general, a la mayoría de las personas, por una razón o por otra, le cuesta mucho el manejo de los números. Esto provoca que diariamente nos puedan manipular con estadísticas y datos sobre economía mundial, salud, paro, precariedad, encuestas electorales, etc. Luego hemos de admitir que sin conocer cómo funcionan ciertas nociones básicas de Estadística somos un blanco perfecto para ser manipulados.

Pero como bien sabemos, las unidades didácticas de Estadística suelen situarse a final del curso lectivo en el que se imparten, lo cual, en numerosas ocasiones, implica la omisión de dichos contenidos curriculares, debido a la escasez de tiempo dentro de la temporalización de los cursos de ciencias.

La nueva normativa, LOMCE, tiene intención de paliar este hecho para poder asegurar que todos los graduados en Bachiller hayan cursado el tema de Estadística y Probabilidad, y lo hace dividiendo los contenidos entre ambos cursos. Como bien sabemos, con la anterior normativa LOE, los contenidos de Estadística y Probabilidad estaban presentes a partir del cuarto curso de la ESO. De ahora en adelante la Estadística Descriptiva se imparte en el primer año de Bachillerato y la Probabilidad en el segundo, recortando así una posible unidad didáctica del temario.

Queremos asegurar por tanto que nuestros estudiantes cursen los contenidos correspondientes de esta rama de las Matemáticas, que es la más aplicada a diario en el mundo de las tecnologías de la información y de la comunicación que habitamos, y cuyos contenidos básicos son necesarios para el correcto desarrollo de muchos de los estudios superiores de formación profesional y carreras universitarias.

Por otra parte, estamos impartiendo clases a alumnos que en su mayoría se encuentran en un rango de edad entre 16-18 años (si consideramos que es la primera vez que cursan la asignatura) y por tanto, si no lo han hecho ya, están a punto de enfrentarse a su mayoría de edad, con todas las responsabilidades que como ciudadanos ello implica.

Debido a lo anterior, el objetivo principal de esta comunicación es el de presentar una serie de actividades relacionadas con la Estadística que permitan desarrollar un pensamiento crítico en los alumnos, tratando de esta manera de motivarles a través de un camino reflexivo y autónomo de la realidad.

El profesor podrá usar estas actividades o bien otras, similares o diferentes, que él mismo plantee, para motivar a sus alumnos, despertar su curiosidad sobre este nuevo conocimiento que se le va a impartir y en suma, predisponerles a este nuevo reto en sus carreras. 
Entre estas actividades que proponemos, se indican en esta comunicación las siguientes: en primer lugar se introducirá la Estadística al alumno a través de su historia. Para ello, se muestra la biografía de una de las mujeres matemáticas más importantes por su relación con la Estadística, si bien no del todo reconocida por la sociedad en general. Hablamos de Florence Nightingale, conocida como la "dama de la lámpara".

Entendemos que el conocimiento de la biografía de Florence puede suscitar el interés de los alumnos por la Estadística, dada la vida tan intensa que llevó gracias a tales conocimientos.

En segundo lugar estableceremos una relación entre la Estadística y los medios de comunicación. Trataremos de llevar a los alumnos a la reflexión a través de graves errores encontrados tanto en prensa como en televisión.

Finalmente, y en tercer lugar, hemos reservado un espacio para acercar la Política al aula a través de su relación con la Estadística.

\section{MOTIVACIONESHISTÓRICAS}

\section{Florence Nightingale}

La dama de la lámpara, como era recordada Florence Nightingale por los soldados heridos en la Guerra de Crimea, utilizó sus conocimientos de Matemáticas y de forma especial, desarrolló la Estadística descriptiva para denunciar la lamentable situación de los hospitales de guerra. A ella se le debe también haber transformado la mala fama de la enfermería en aquella época, asociada a mujeres de clase baja, desaliñadas e inútiles, en una profesión respetada y respetable para las mujeres.

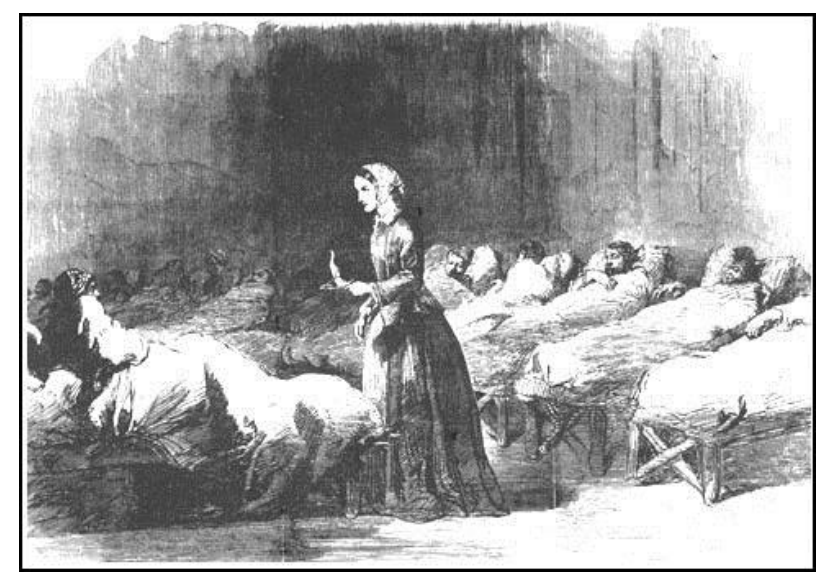

Figura 1. La dama de la lámpara.

Florence Nightingale nació circunstancialmente en Florencia (de ahí su nombre), en el Gran Ducado de Toscana, el 12 de mayo de 1820 y murió en Inglaterra el 13 de agosto de 1910. Su familia, británica, había heredado de un pariente rico, por lo que llevaba una vida acomodada pasando largas 
temporadas en el campo o de viaje por Europa. Según sus palabras, en medio de aquella vida su conciencia permanecía alerta a esa otra sociedad que vivía en la pobreza, marginada y alimentándose de los desperdicios de la suya.

Florence fue educada por su padre, familiarizándose así con los clásicos, con la Biblia y con temas políticos. Ya en su juventud pidió a sus padres que la dejaran estudiar Matemáticas, cosa poco habitual para una mujer en aquella época, a lo que sus padres se resistieron (Mataix, 1999). Finalmente contó con dos grandes matemáticos como profesores, Sylvester y Cayley. En esta etapa de su juventud sintió la vocación a la enfermería. No se casó, aunque no por falta de pretendientes, y dedicó su larga vida a esa misión que ella entendió que era una llamada de Dios.

A pesar de la oposición de su familia, pasó por distintos hospitales para adquirir experiencia como enfermera y el 4 de noviembre de 1854 llegó a un suburbio de Estambul. Allí logró reunir a 38 enfermeras para hacerse cargo del hospital de guerra de Escutari. Había comenzado la Guerra de Crimea, un conflicto que enfrentaba a Gran Bretaña, Francia y Turquía contra Rusia. Fue la primera vez que un periódico, The Times, mandaba un corresponsal de guerra, que relataba el conflicto mediante el telégrafo, y la primera vez que se tenían fotos. La opinión pública británica fue adquiriendo una actitud cada vez más crítica porque quedó patente la ineficacia y corrupción de los mandos militares. La situación del hospital al que llegó Florence era un desastre: barracas llenas de heridos, pulgas, piojos y ratas; fosas sépticas y emanaciones cerca de las barracas; mala alimentación y agua fría para la lavandería y la desinfección. La probabilidad de morir en el hospital era siete veces superior a la de morir en el campo de batalla, ya que eran las enfermedades contagiosas como el tifus o el cólera las que causaban mayores estragos. Ante la poca atención que recibió Florence de los mandos militares, usó sus contactos en el Times para denunciar la situación. Calculó la tasa de mortalidad en el hospital, que cuando llegó estaba por encima del 60\%, recolectó los datos y organizó un sistema para llevar un registro. Su formación matemática hizo que no pasara por alto la elevada tasa de mortalidad y que buscara las causas. Usó su propio dinero para establecer una fuente de agua potable, comprar frutas, verduras y equipamiento sanitario, consiguiendo reducir en poco más de un año la tasa al $2 \%$.

Florence usó toda la información para crear unos diagramas, que en la actualidad son de uso común, llamados polares o gráficos radiales, donde representaba en cada cuña coloreada las cifras de mortalidad durante la guerra, de 1854 a 1856 . Como a ella le gustaba decir, en palabras de Goethe, "el mundo está gobernado por los números porque son ellos los que nos dicen si está bien o mal gobernado". Con ellos logró llamar la atención de la reina Victoria y la del primer ministro para llevar a cabo una reforma sanitaria. 


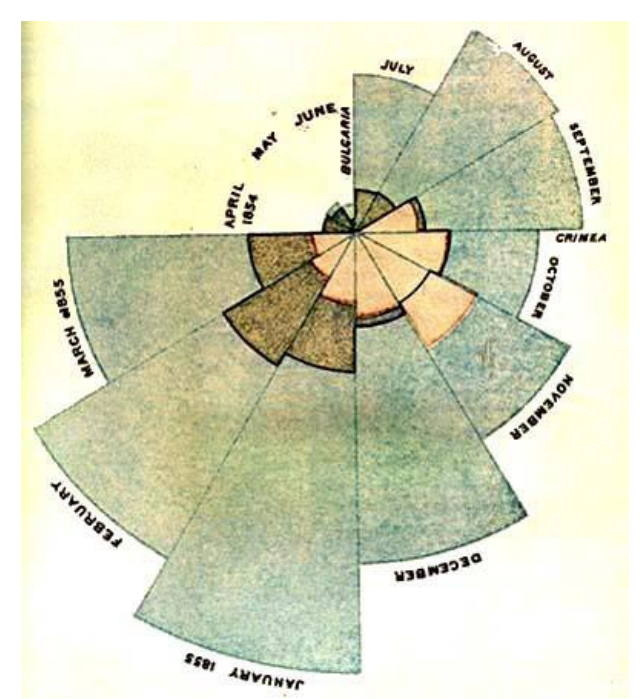

Figura 2. Diagrama Polar.

Aplicó sus conocimientos de Estadística a la epidemiología y a la Estadística sanitaria. Fue la primera mujer admitida en la Royal Statistical Society británica, y miembro honorario de la American Statistical Association.

A la edad de 36 años, Florence era una figura famosa y respetada en todo el mundo. No obstante, declinó todo tipo de honores públicos afirmando que la mejor recompensa a sus servicios sería el nombramiento de una comisión que investigara el estado de la asistencia médica en el ejército. Y escribió: "en Crimea unos nueve mil soldados reposan en tumbas olvidadas, muertos por causas que pudieron prevenirse".

Florence sentó las bases de la profesionalización de la enfermería al fundar en 1860 su escuela de enfermería en el hospital Saint Thomas de Londres, actualmente parte integrante del King's College de Londres y del NHS (fue la primera escuela laica de enfermería en el mundo).

Su trabajo fue la fuente de inspiración de Henri Dunant, fundador de la Cruz Roja y autor de las propuestas humanitarias adoptadas por la convención de Ginebra. Asimismo, escribió varios libros sobre la profesión que fueron utilizados durante mucho tiempo como manuales de enseñanza de la enfermería en numerosos países. 


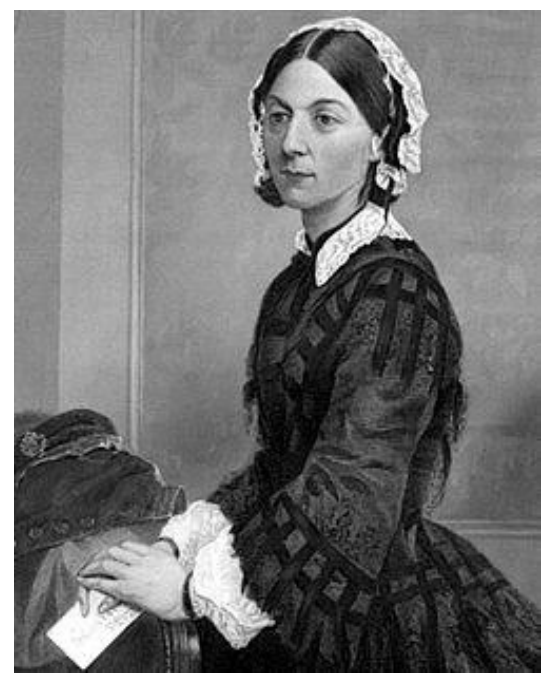

Figura 3. Florence Nightingale.

Los quince últimos años de su vida los pasó ciega y postrada en cama debido a unas fiebres contraídas en Crimea. Fue innovadora en la recolección, tabulación, interpretación y presentación gráfica de las estadísticas descriptivas, demostrando que un fenómeno social puede ser medido objetivamente $\mathrm{y}$ analizado matemáticamente. En 1858 fue elegida miembro de la Sociedad Estadística de Inglaterra, siendo la primera mujer en entrar en dicha institución, también miembro honorífico de la American Statistical Association en 1874, y en 1883 la reina Victoria le otorgó la Cruz Roja Real por su labor. También fue la primera mujer en recibir la Orden de Mérito del Reino Unido por el rey Eduardo VII, en 1907. En 1908, le fueron otorgadas las Llaves de la Ciudad de Londres.

El monumento a Crimea fue erigido en 1915 en Waterloo Place, en Londres, para honrar la contribución que hizo Florence Nightingale a los soldados de la Guerra de Crimea.

El Juramento Nightingale efectuado por los enfermeros al graduarse, fue creado en su honor en 1893. El Día Internacional de la Enfermería se celebra en la fecha de su cumpleaños.

Florence falleció en Londres, en agosto de 1910. Puede obtenerse mayor información sobre su biografía en (web1).

\section{Actividad 1}

Una vez presentada en clase la biografía de Florence podría proponerse la siguiente actividad para que los alumnos realizasen un pequeño trabajo de investigación:

a) Completa la biografía que se ha comentado en clase con nuevos datos que encuentres en otras fuentes (bibliográficas, webs, revistas, etc.) a las que puedas acceder. 
b) Busca la biografía de otro de los muchos personajes relevantes relacionados con la Estadística y que hayan contribuido de manera notable al desarrollo de esta disciplina.

Este trabajo se puede programar de forma individual o en pequeños grupos de trabajo, de forma que se expongan las biografías trabajadas al resto de la clase una vez terminadas.

\section{ESTADÍSTICAS Y MEDIOS DE COMUNICACIÓN}

Una vez vista la importancia que tuvieron los medios de comunicación en la guerra de Crimea sería buen momento para relacionarlos con la Estadística. Entre otras cosas, gracias a ellos se tuvo conocimiento del absoluto desastre en el que se encontraban los hospitales militares, por tanto es obvia la importancia de los medios de comunicación en nuestra sociedad. A pesar de ello, es un área donde con mayores errores en el uso de la Estadística nos podemos encontrar. Con frecuencia los profesionales de la información caen o se dejan caer en todo tipo de errores; unos leves y otros que no lo son tanto.

Un caso frecuente lo encontramos cuando un medio de comunicación se deja llevar por el sensacionalismo y utiliza las frecuencias relativas y absolutas a su antojo.

Un ejemplo de titular para este caso: "El puente del Pilar deja 15 muertos en las carreteras" (Diario El Mundo, 13/10/2015). Esta noticia vista en términos relativos no nos sorprendería de la misma manera ni tendría el mismo impacto. De hecho, en términos proporcionales tenemos aproximadamente el mismo número de víctimas que en otro periodo de cuatro días. Para ello tenemos que tener en cuenta el gran número de traslados a los que se ven sometidos las carreteras durante esas fechas. Pero entonces nos quedaríamos sin una noticia que vender. Un titular alternativo podría ser: "El puente del Pilar deja la misma proporción de muertos en las carreteras que cualquier periodo de cuatro días del año.”

Esta picaresca se suele emplear tanto en un sentido como en el otro. Es decir, se toman medidas absolutas o relativas según intereses o quizás por vicios tomados desde el pasado. Por ejemplo: "Los homicidios en la provincia aumentaron un $60 \%$ respecto al pasado año." Si en este caso, el medio de comunicación en lugar de darnos los datos relativos nos hubiera dado el titular con datos absolutos, el titular habría perdido toda la fuerza "Los homicidios en la provincia pasaron de 5 a 8 en el último año".

Sin embargo, tanto en el caso de los accidentes de tráfico como en el de los homicidios no podemos culpar al medio de comunicación de mentirnos. 
Otro caso de malinterpretación de la Estadística proviene de curiosidades que los medios convierten en noticias. "Recientemente nos hemos enterado de la increíble historia de una pareja en Inglaterra que sin planearlo recibieron a sus

3 hijos exactamente a la misma hora: 7:43. Expertos comentan que la probabilidad que esto suceda es de 1 en 300 millones." (blog.myheritage.es). Es obvio que es una gran casualidad que esto ocurra. Pero si tenemos en cuenta que la población mundial supera los 7000 millones de habitantes, nos encontramos ante la certeza de que no es raro que esto ocurra, sino de que lo normal es que ocurra, incluso en varias ocasiones o familias. No diferenciamos entre la probabilidad de que un suceso ocurra con la probabilidad de que ese suceso nos ocurra a nosotros.

Al respecto de lo anterior, proponemos las siguientes actividades a realizar por los alumnos.

\section{La paradoja del cumpleaños}

Si estuvieras en una reunión de 23 personas, ¿qué es más probable, que haya dos personas que compartan fecha de cumpleaños (día y mes) o que no?

Esta pregunta da lugar a la paradoja del cumpleaños, la cual establece que si hay 23 personas reunidas hay una probabilidad del 50,7\% de que al menos dos personas de ellas cumplan años el mismo día (Ruiz, 1999). Incluso si aumentamos la reunión a 57 personas o más, la probabilidad se dispara por encima del 99\%.

Esta paradoja nos muestra que incluso sucesos que se nos antojan improbables ocurren. De hecho, lo normal es que ocurran; y eso nos cuesta diferenciarlo.

\section{Actividad 2}

a) Ahora que conocemos la Paradoja del Cumpleaños, ¿cuál es la probabilidad de que al menos dos personas de la clase tengan el mismo día de cumpleaños?

b) ¿Cuál es el número mínimo de personas necesario en la clase para que sea más probable encontrar al menos dos con el mismo día de cumpleaños que no encontrarlas?

c) ¿Debemos tomar todo lo que nos llega como verdadero e inamovible o sin embargo debemos ser impermeables y críticos con determinadas informaciones que nos llegan de los medios?

\section{Actividad 3: ¿Nos mienten los medios?}

Los gráficos son muy útiles, ya que con un simple vistazo podemos visualizar muchísima información. Pero en vista a que tendemos a creernos más los gráficos que la información escrita, estos se pueden convertir también en peligrosas armas de manipulación. Es decir, a menudo olvidamos que esta información visual puede contener errores y, por supuesto, ser tendenciosa. 
La siguiente actividad comprende contenidos de estadística unidimensional presente en los cursos de secundaria, aunque el estudio de la tendenciosidad es sólo parte de los contenidos de $4^{\circ}$ de la ESO y de Bachillerato. No olvidemos que dentro de la nueva normativa LOMCE encontramos contenidos de las asignaturas de Matemáticas orientadas a las enseñanzas académicas y a las enseñanzas aplicadas de $4^{\circ} \mathrm{ESO}$ que especifican: "Análisis crítico de tablas y gráficas estadísticas en los medios de comunicación”. Y por último en las cuatro asignaturas de Matemáticas de Bachillerato el siguiente criterio de evaluación: "interpretar de forma crítica informaciones estadísticas presentes en los medios de comunicación, la publicidad y otros ámbitos, detectando posibles errores y manipulaciones tanto en la presentación de los datos como de las conclusiones”.

Se trata de una actividad de aprendizaje puesto que los estudiantes ya conocen los contenidos matemáticos que necesitan aplicar para desarrollarla. Podría servir como repaso de contenidos estadísticos (unidimensionales) o como actividad de introducción y motivación para los cursos de Bachillerato, ya que implican el análisis de datos en los medios de comunicación, algo con lo que nuestros alumnos están muy familiarizados.

Con esta actividad estamos trabajando la competencia en comunicación lingüística, mediante el debate argumentado en clase; la competencia social y cívica, ya que se trabajan temas de relevancia social como es la manipulación de los medios de comunicación; la competencia de aprender a aprender, puesto que desarrollamos la conciencia crítica del alumnado, buscando que las nuevas generaciones sean capaces de detectar cualquier tipo de tendenciosidad dentro de la información que reciben; y por último, la competencia matemática, al estar trabajando con datos estadísticos.

Se reparten una serie de gráficas extraídas de medios de comunicación españoles para analizar su tendenciosidad y manipulación. De nuevo dividimos a los estudiantes en grupos, en esta ocasión cada grupo recibe una de las siguientes tres imágenes, extraídas de diferentes medios de comunicación: 


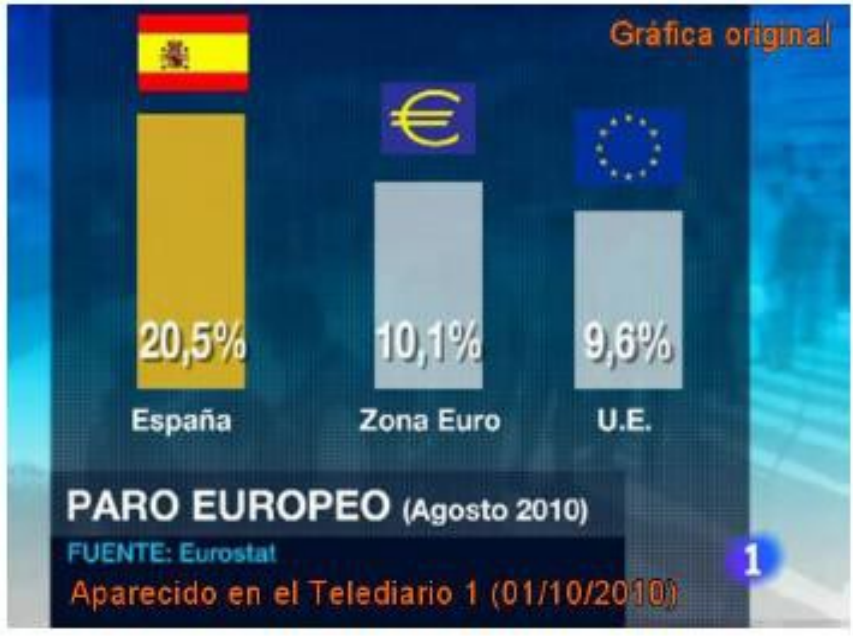

Figura 4. Paro europeo. (Fuente: TVE)

\section{¿Quién ha ganado el debate?}

Marivo, 28 do Junbe de 2011

Rajoy: 831 votos $(20,2 \%)$

Zapatero: 3551 votos (86,2\%)

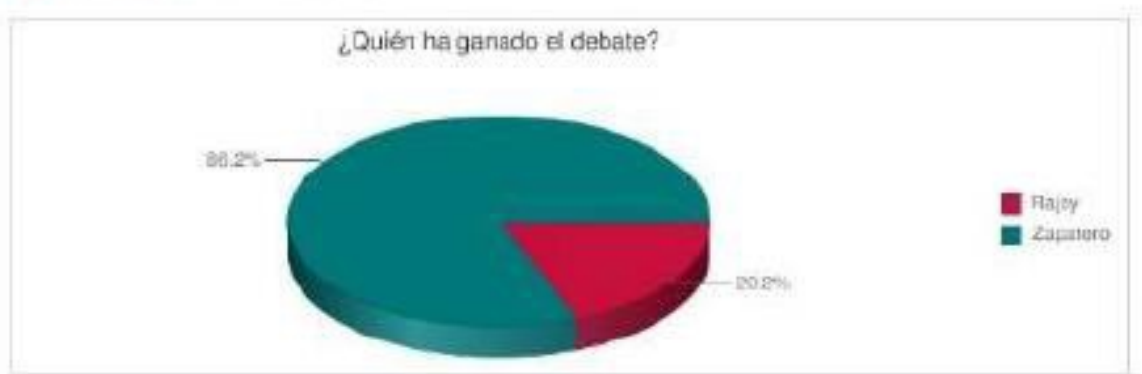

Figura 5. ¿Quién ganó el debate? (Fuente: El País)

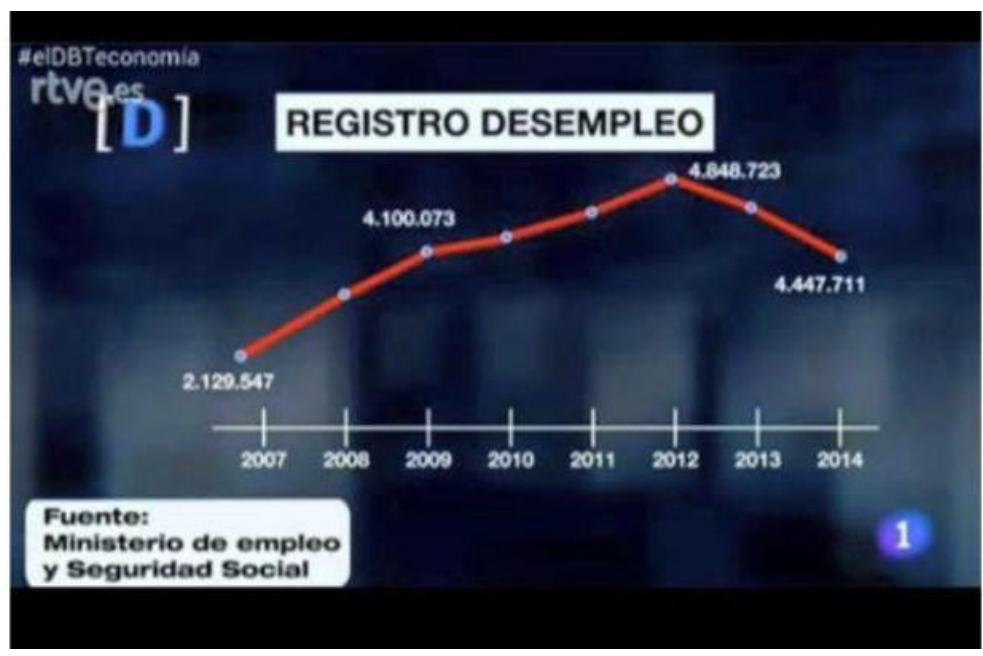

Figura 6. Registro de desempleo. (Fuente: TVE) 
Posteriormente tienen que realizar un trabajo donde respondan a las siguientes cuestiones:

a) ¿Cómo son los datos representados, cualitativos o cuantitativos? ¿Discretos o continuos? Razonar la respuesta.

b) ¿A qué tipo de gráfica estadística corresponde? ¿Qué otros tipos de gráficos conocéis para representar este tipo de datos?

c) ¿Qué errores veis en la gráfica? (Errores de agrupación de datos, de área o de escala...)

d) Con los datos aportados por la imagen realizad una representación rigurosa de los mismos en el mismo tipo de gráfico estadístico y en otro distinto de los expuestos en el punto 2.

Para concluir, uno de los grupos de cada tipo de gráfica deberá exponer el trabajo al resto de sus compañeros e iniciar un debate con el resto del aula sobre la tendenciosidad de la imagen y las consecuencias de su lectura errónea, bajo la moderación del profesor o profesora (existen en la literatura bastantes textos sobre esta temática. Pueden consultarse (Campbell, 2002; Corbalán, 2007 y Sowell, 2013)).

\section{POLÍTICA Y ESTADÍSTICA}

El mundo de la política es uno de los que más se lucra del manejo de la estadística e incluso de su manipulación. La tergiversación de datos estadísticos y su manejo para conseguir fines determinados no es siempre obra de los profesionales de la estadística según Darrell Huff (2002). Cualquier estudio estadístico puede verse cambiado, exagerado, simplificado y tergiversado al tomar solo una parte conveniente del mismo.

Para este caso, el trabajo de los autores se ha centrado en la elaboración de una serie de actividades para el curso de $1^{\circ}$ de Bachillerato relacionadas con la política. Se elige este curso académico por ser en el que mayor peso tiene la Estadística Descriptiva dentro de todo el currículo de la educación secundaria, aunque muchas de las actividades que se desarrollarán a continuación pueden ser llevadas a cursos inferiores de la secundaria.

Uno de los recursos utilizados en estas actividades para fomentar el uso de las TIC's es la consulta de bases de datos de organismos oficiales del Estado encargados de realizar los censos y estadísticas de interés nacional

\section{Actividad 4: El sistema electoral}

Con esta actividad, pudiendo ser usada como actividad de introducción o como repaso de conocimientos previos, trabajamos los parámetros estadísticos así 
como las competencias: lingüística, mediante la lectura y la redacción; social y cívica, ya que se comentan distintos modelos democráticos por países y se pondrá en relevancia la importancia del respeto de la libertad de opinión y voto; aprender a aprender, ya que los propios alumnos deben realizar las búsquedas necesarias para responder a las preguntas planteadas mediante la investigación; y la matemática, al estar trabajando con datos estadísticos.

Los alumnos deben leer el siguiente texto, meditarlo e ir respondiendo a las siguientes cuestiones:

a) ¿Cómo habéis elegido al delegado de la clase?

Tal vez se hizo una votación en la que cada alumno elegía a un compañero y el más votado fue nombrado delegado. Tal vez en cada papeleta se podían anotar un máximo de dos candidatos. Quizás se hizo una segunda vuelta para elegir entre los dos más votados.

b) Hay un parámetro estadístico que designa al candidato más votado. ¿Cuál es?

Hay muchas maneras de realizar una votación. Por ejemplo, para elegir la ciudad que organizará los Juegos Olímpicos se vota primero entre las ciudades candidatas y se elimina la que menos votos haya conseguido; se vuelve a votar y se retira de nuevo la que menos votos reciba; así hasta quedarse con la ciudad ganadora.

Los sistemas electorales para elegir a los gobernantes también son variados según los países. El sistema electoral español no es proporcional al número de votos; sigue la llamada ley D'Hondt. En otros países, como en Brasil, después de la primera elección puede realizarse una segunda vuelta en la que compiten los dos candidatos que más votos recibieron si ninguno de ellos obtuvo el $50 \%$.

c) ¿En qué otros países se realizan la segunda vuelta? ¿Para qué sirve?

La Estadística ha estudiado en profundidad cuál es el sistema electoral más justo y ha llegado a una conclusión: no hay un sistema electoral idóneo, todos tienen algún inconveniente que deja insatisfechos a algunos, y además dan lugar a situaciones paradójicas. Por ejemplo, en una primera vuelta pueden quedar ordenados los candidatos así: $A>B>C$, y en la segunda vuelta los que votaron a $C$ se decantan mayoritariamente por B y gana este.

En nuestro sistema electoral se ha dado el caso de que el partido con más votos no sea el que más escaños recibe.

d) Buscad al menos un caso en el que haya pasado esto.

e) ¿Qué significa mayoría simple y mayoría absoluta? 
f) En casi todas las votaciones el voto es secreto. ¿A qué crees que se debe?

\section{Actividad 5: Variables estadísticas}

Se trata de una actividad de enseñanza-aprendizaje, ya que impartimos nuevos conceptos a los estudiantes a la vez que ellos los aplican directamente a su trabajo en equipo.

Esta actividad es aplicable a $4^{\circ}$ de ESO y $1^{\circ}$ de Bachillerato. Si estamos en Bachillerato los alumnos vienen de estudiar estos contenidos en la ESO, por lo cual podría considerarse como una actividad de repaso para afianzar los conocimientos previos. Aunque como comentamos en la introducción, es posible que alguno de estos parámetros no se hayan estudiado en secundaria, siendo en ese caso, al igual que para $4^{\circ}$ ESO una actividad de aprendizaje. Trabajamos la competencia en comunicación lingüística, mediante la redacción y exposición oral al resto de compañeros; la competencia social y cívica, ya que se trabaja sobre el concepto de democracia y ciudadanía; la competencia de aprender a aprender, ya que los propios alumnos deben realizar las búsquedas necesarias para responder a las preguntas planteadas; la competencia digital, ya que necesitan de la búsqueda por internet y el manejo de la página web del Instituto Nacional de Estadística; y la competencia matemática, al estar trabajando con datos estadísticos.

Tras un repaso del concepto de variable estadística, así como de las diferencias entre variables cualitativas, cuantitativas discretas y cuantitativas continuas, el para qué sirven las muestras de población, las clases o intervalos y las marcas de clase, repasaremos también los distintos parámetros: frecuencias relativa, absoluta y acumulada, media aritmética, moda, mediana, rango, varianza y desviación típica de la siguiente manera.

Para repasar todos estos parámetros los estudiantes deben realizar tres estudios estadísticos distintos donde deben: en primer lugar calcular todas las variables expuestas en sus cuadernos, en segundo lugar y con la ayuda de una tabla estadística en Calc (o cualquier otra hoja de cálculo que posea el centro) corregir posibles fallos y por último presentar una gráfica que muestre los datos estudiados (diagrama de barras, histograma, etc.).

Para ello la clase se divide en grupos de trabajo. El docente facilitará una copia de los datos a cada grupo para poder realizar el trabajo al comienzo de la siguiente sesión. Cada grupo debe realizar los siguientes trabajos en base a uno de los temas a elegir por sorteo:

- Variables cualitativas: partidos políticos votados en España en las últimas elecciones generales www.interior.gob.es/informacion-electoral. Presidentes de partidos políticos entrevistados en la Radio Televisión Española en 2015. Los partidos y presidentes se nombrarán siguiendo las letras del alfabeto de modo que permanezcan anónimos. Los 
partidos se representan con letras mayúsculas y el presidente que corresponda a dicho partido tendrá la misma letra en minúscula.

- Variables cuantitativas discretas: número de escaños de cada partido en las últimas elecciones generales www.interior.gob.es/informacion- electoral y número de millones de euros defraudados en los casos de corrupción por cada partido (incluir como mínimo tres partidos de manera anónima).

- Variables cuantitativas continuas: inversión de los últimos dos gobiernos de España en Educación e inversión de los últimos dos gobiernos de la comunidad autónoma en Educación.

Evidentemente cada grupo tendrá una mezcla de los tres tipos de variables y necesitará del trabajo de los otros grupos para poder completar sus datos. Cada grupo expondrá su trabajo al resto de la clase al final de la actividad.

\section{Actividad 6: Sistema electoral y circunscripciones.}

En la siguiente actividad seguimos trabajando de manera cooperativa por grupos. Trabajamos la competencia en comunicación lingüística, mediante el debate argumentado en clase; la competencia social y cívica, ya que se trabajan temas de relevancia social como el sistema electoral y las circunscripciones; y la competencia matemática al seguir trabajando con datos estadísticos.

El trabajo de cada grupo no solo consiste en realizar la siguiente actividad sino que también deberán exponerla en la última sesión al resto de la clase. Los alumnos deben leer el siguiente texto e ir respondiendo de manera reflexiva a las preguntas que se encuentran en el:

El sistema electoral español, definido en la Constitución de 1978, está diseñado para favorecer a las mayorías y así obtener gobiernos más estables. Siendo esto así, ¿cómo explicaríais que en las elecciones de noviembre de 2015 no se pudiese formar gobierno?

Esto se consigue a través del sistema D’Hondt, un método para repartir los escaños de modo aproximadamente proporcional al número de votos que ha obtenido cada una de las candidaturas. Es utilizado en muchos países. El sistema es el siguiente: para repartir $N$ escaños, una vez escrutados todos los votos, se calculan una serie de cocientes mediante la expresión V/n, donde $V$ representa el número total de votos recibidos por cada uno de los partidos y $n$ son los números enteros desde 1 hasta $N$. La asignación de escaños se hace tomando los $N$ mayores cocientes.

Por ejemplo, en una localidad hay cuatro partidos: $A, B, C$ y $D$. El número de escaños es $N=10$. Al dividir el número total de votos entre $1,2,3, \ldots, 10$ se obtiene la Tabla 1. 
Tabla 1. Reparto de escaños con el sistema D’Hondt.

\begin{tabular}{|l|c|c|c|c|}
\hline Partido & $\mathrm{A}$ & $\mathrm{B}$ & $\mathrm{C}$ & $\mathrm{D}$ \\
\hline Votos & 840 & 1060 & 360 & 400 \\
\hline Votos $: 2$ & 420 & 530 & 180 & 200 \\
\hline Votos $: 3$ & 280 & 353 & 120 & 133 \\
\hline Votos $: 4$ & 210 & 265 & 90 & 100 \\
\hline Votos $: 5$ & 168 & 212 & 72 & 80 \\
\hline Votos $: 6$ & 140 & 177 & 60 & 67 \\
\hline$\ldots$ & $\ldots$ & $\ldots$ & $\ldots$ & $\ldots$ \\
\hline Votos $: 10$ & 84 & 106 & 36 & 40 \\
\hline
\end{tabular}

Las celdas sombreadas corresponden a los escaños asignados. El partido $B$ ha obtenido 5 escaños; el A ha obtenido 3, y el C y el D, 1 cada uno.

a) En unas elecciones generales en una provincia, los resultados de los cinco partidos más votados fueron:

Tabla 2. Votos por partido.

\begin{tabular}{|l|c|c|c|c|c|}
\hline Partido & A & B & C & D & E \\
\hline Votos & 230728 & 194511 & 114783 & 27374 & 19484 \\
\hline
\end{tabular}

Entre estos, los 16 partidos restantes, los votos nulos y en blanco completaron un total de 624432 votos.

- Determinad el porcentaje de votos que correspondió a cada uno de estos cinco partidos.

- $\quad$ Representad en un diagrama de sectores los datos aportados.

b) Si en esta provincia se adjudicaban 8 diputados, aplicad el sistema D'Hondt para determinar cuántos diputados correspondieron a cada partido.

c) Representad en un nuevo diagrama de sectores la distribución del número de diputados que corresponde a cada partido.

d) Comparad el porcentaje de votos con el de diputados obtenidos. ¿Qué concluís?

e) En otra provincia, el partido X obtuvo 15 diputados con 1377996 votos, y el $Y$, con 1723370 votos, obtuvo 18. Comparad el número de votos por diputado de cada partido entre las dos provincias. 
f) Abrid un debate en la clase en el que cada uno exponga su opinión sobre el sistema electoral español. ¿A qué conclusiones llegáis?

g) Debatid si son justas o no las circunscripciones realizadas en España argumentado vuestras opiniones.

\section{CONCLUSIONES}

En esta comunicación se han propuesto diversos ejemplos de actividades sobre historia, política y medios de comunicación conjugadas junto a la Estadística. A través de ellas se puede trabajar con los alumnos sobre cuestiones de actualidad de la vida real. A su vez, podemos aprovechar el perjuicio que ocasionan los errores o las manipulaciones que se hacen con la Estadística y que llegan hasta nosotros casi sin que nos demos cuenta. De esta manera no solo corregimos sino que acercamos la realidad de nuestro entorno al aula.

Nuestra intención era la de haber llevado a la práctica esta unidad en alguno de los centros de Secundaria y Bachillerato a los que uno de los autores de esta comunicación viene acudiendo de forma regular (una o dos veces por curso) desde hace ya algunos años, como parte de la fase de prácticas de la investigación sobre la enseñanza de las Matemáticas en los IES que se lleva a cabo en el grupo de investigación que dirige, al objeto de analizar y contrastar sus resultados.

Sin embargo, por razones de calendario de esos centros y del tiempo que supondría llevar a la práctica estas actividades, este último deseo no se ha podido materializar todavía, motivo por el cual aún no podemos presentar ningún análisis de resultados.

En todo caso, los autores pensamos que:

1.- Sería necesario incluir algunas nociones de historia de las matemáticas, medios de comunicación y Política durante los cursos de Secundaria y Bachillerato. De este modo no solo enriqueceríamos los contenidos sino también los conocimientos del alumnado.

2.- Las actividades que proponemos son una buena forma de acercar la Estadística a estudiantes ya cercanos a la edad adulta. Plantear problemas cercanos al día a día motivará la reflexión autónoma y el análisis crítico de las circunstancias por parte del alumno.

3.- Es fundamental trabajar a través de las matemáticas una de las competencias claves determinadas por la ley educativa: la competencia cívica. Esta se basa en el conocimiento crítico de los conceptos de democracia, justicia, igualdad, ciudadanía y derechos humanos y civiles, por lo cual se hace 
necesaria la incorporación de contenidos de índole política a las aulas de Secundaria y Bachillerato.

4.- Dotaríamos al alumno de una mayor libertad de pensamiento, de forma que sus decisiones no se vean influidas y/o manipuladas por los medios de comunicación. A su vez, fomentaríamos que los alumnos se incorporen al derecho al voto con plena conciencia de la importancia que ello conlleva.

Por todo ello, reiteramos nuestra intención de llevar a la práctica esta propuesta, analizar los resultados obtenidos e incentivar al profesorado de los centros a realizar actividades en sus aulas de este mismo tipo o similares.

\section{REFERENCIAS}

Campbell, S. K. (2002). Flaws and Fallacies in Statistical Thinking. Denver: University of Denver.

Corbalán, F. (2007). Matemáticas de la vida misma. Barcelona: Editorial Graó, de IRIF, S.L.

Huff, D. (2002). Cómo mentir con estadísticas. Brasil: Editorial Ridendo Castigat Mores.

Mataix, S. (1999): Matemática es nombre de mujer. Barcelona: Editorial Rubes.

Ruiz, G. (1999). La paradoja de San Petersburgo. Una reivindicación didáctica. En Revista SUMA, (32), 5-9.

Sowell, T. (2013). La Economía: verdades y mentiras. Barcelona: Planeta De Agostini Profesional y Formación, S.L.

[web1] http://www-history.mcs.st-andrews.ac.uk/history/Biographies

(web sobre biografías de matemáticos). Consultado, 20 de junio de 2016. 
